\title{
¿On the Importance of Statistical Homogeneity to the Scaling of Rain
}

\author{
A. R. JAMESON \\ RJH Scientific, Inc., El Cajon, California
}

(Manuscript received 11 September 2018, in final form 13 February 2019)

\begin{abstract}
Scaling studies of rainfall are important for the conversion of observations and numerical model outputs among all the various scales. Two common approaches for determining scaling relations are the Fourier transform of observations and the Fourier transform of a correlation function using the Wiener-Khintchine (WK) theorem. In both methods, the observations must be wide-sense statistically stationary (WSS) in time or wide-sense statistically spatially homogeneous (WSSH) in space so that the correlation function and power spectrum form a Fourier transform pair. The focus here is on developing an explicit understanding for the requirement. Statistically heterogeneous (either in space or time) data can produce serious scaling errors. This work shows that the effects of statistical heterogeneity appear as contributions from cross correlations among all of the distinct contributing rainfall components using either method so that the correlation function and its FFT do not form a transform pair. Moreover, the transform then also depends upon the time and location of the observations so that the "observed" power spectrum no longer represents a "universal" scaling function beyond the observations. An index of statistical heterogeneity (IXH) defined in previous work provides a way of determining whether or not a set of rain data may be considered to be WSS or WSSH. The greater IXH exceeds the null, the more likely the derived power spectrum should not be used for general scaling. Numerical simulations and some observations are used to demonstrate all of these findings.
\end{abstract}

\section{Introduction}

Observations and numerical models produce results on many different usually incompatible scales from fractions of a meter up to kilometers. How can one then incorporate observations from, say, those of a single rain gauge into forecast models often having resolutions on the order of kilometers? Likewise, how can the products from these numerical models be downscaled to fractions of a kilometer for comparisons, say, with a network of rain gauges? Hence, scaling is an important topic in many rainfall studies (e.g., Berndtsson and Niemczynowicz 1988; Ochoa-Rodriguez et al. 2015; de Lima et al. 2012).

These objectives are often approached using power spectra, which makes it possible to scale over a range of dimensions. There are different methods for deriving such power spectra. One is to take the direct Fourier transform of observations and then to compute a power spectrum by multiplying this transform by its complex

Denotes content that is immediately available upon publication as open access.

Corresponding author: A. R. Jameson, arjatrjhsci@verizon.net conjugate. This is the so-called direct Fourier transform (DFT) method. An alternative route to the power spectrum that may be statistically superior is to take the Fourier transform of correlation functions using the Wiener-Khintchine (WK) theorem (Wiener 1930; Khintchine 1934). The latter shows that the correlation function can be decomposed into its spectral components (power spectrum) using a Fourier transform such that as the lags of the correlation function decrease, the corresponding wavelengths of the Fourier components contributing to the function increase. The "power" or magnitude of each component is equivalent to the zero-mean variance contribution at each particular wavelength. Specifically, for real valued functions and in terms of time, the autocorrelation function of a variable $Y$ can be expressed as

$$
\rho(t)=\int_{-\infty}^{\infty} Y(t) Y(t+\tau) d \tau
$$

for delay $\tau$. What the WK theorem shows is that the Fourier transforms

$$
\rho(t)=\int_{-\infty}^{\infty}|Y(f)|^{2} \exp (2 \pi i f t) d f
$$


and

$$
S(f)=|Y(f)|^{2}=\int_{-\infty}^{\infty} \rho(t) \exp (-2 \pi i f t) d t
$$

form a Fourier transform pair so that one can be converted into the other, where $S(f)$ is the so-called power (variance) spectrum and $i$ represents the imaginary number. The same applies to spatial calculations as well.

Regardless of methodology, however, any valid power spectrum can only be defined under conditions of statistical homogeneity; that is, the mean value and variance remain constant in time [wide-sense statistical stationarity (WSS)] or constant in space [wide-sense statistically homogeneous (WSSH)]. Under these conditions, the Fourier transform of a correlation function and the variance or power spectrum form a Fourier transform pair whether using the DFT approach or the WK theorem. Another version of this general approach has been developed recently for the numerical simulation of nonstationary and spatially heterogeneous datasets (defined below) using a short-spaced Fourier transform (SSFT) (Nerini et al. 2017). In addition, insofar as one accepts the multifractal characterization of rain and insofar as one is willing to ignore the effects of advection on the measurements, Gires et al. (2014) provide an example of power-spectral downscaling in order to compare radar and rain gauge measurements at the same spatial scale.

The importance of statistical stationarity is emphasized by many investigators such as Koutsoyiannis and Montanari (2015) and Montanari and Koutsoyiannis (2014) and others. Here we note that observations are realizations of a statistical and physical process having defined characteristics. While it is the process that possesses the statistical characterizations such as statistical homogeneity in time or space, an observer usually has access to only one realization of a statistical process visà-vis a set of observations. It is, therefore, usually assumed explicitly or implicitly that the data reflect the statistical characteristics of the process itself so that we may speak about the statistical characterization of the data directly since that is all the information usually available. This is done here as well. Furthermore, whether a series of observations may be considered to be stationary or not will depend upon the length of the observations. On one extreme some argue that because of climate change, nothing is stationary (Milly et al. 2008). This is clearly an exaggeration when considering only, say, a few hours of data when the rate of climate change is negligible compared to the variability of the rainfall itself. On the other hand there are those who consider stationarity still to be a vital assumption for the analyses of hydrological data (Matalas 2012). The purpose here is to offer an approach for detecting when nonstationarity may be a problem especially in relatively short-term observations of rainfall rates.

In this paper, therefore, and in contrast to previous work analyzing real data, the focus here is on developing an explicit understanding of why WSS (WSSH) is required for the correct application of the WK theorem. Specifically, we examine in detail why the thoroughly established WSS (WSSH) requirement is necessary for generating a valid power spectrum by examining the Fourier transform between the correlation function and its Fourier transform. In the DFT approach, a series of observations is Fourier transformed to yield a variance spectrum. This spectrum can then be inverse Fourier transformed to yield a correlation function. To be a transform pair, however, the Fourier transform of this correlation function must then again yield the same spectrum. This will be the case if the data are WSS (WSSH). Whether one determines the FFT spectrum first or the correlation function first, all valid power spectra must still satisfy this transform pair requirement; that is, they must satisfy the WK theorem. What is new here is not the well-established requirement of WSS (WSSH) for valid power spectra, but rather a more detailed and hopefully illuminating exploration of the mathematics of why this is so. In this work, this is achieved using numerical simulations so that conditions can be controlled explicitly. In doing so, we use the technique developed in Jameson et al. (2018). Three applications to real data are briefly illustrated in the appendix, but many more examples may be found in Jameson et al. (2018).

Of course, many investigators have calculated variance spectra via the WK theorem for various scales in rain (e.g., Crane 1990; Kiely and Ivanova 1999) using the Fourier transform of the relatively easily observed autocorrelation function. Some investigators are well aware of the need for WSS or WSSH (e.g., Pegram and Clothier 2001; Sinclair and Pegram 2005; Krajewski et al. 2003), but many others have not, so that the interpretation of their findings is arguable particularly since many of them are based upon radar observations, which have their own filtering effects (Jameson 2017). However, the WK theorem provides a convenient route to the power spectrum from observations, while, after proper filtering, the power spectra can, in turn, yield autocorrelation functions useful for interpreting measurements collected over finite temporal or spatial domains of different sizes including networks and by remote sensors (Jameson 2017).

As discussed above, however, the validity of the autocorrelation function and the variance spectrum forming 
a Fourier transform pair depends upon the data being WSS (WSSH) (Wiener et al. 1997). In rain, strict sense stationarity (constancy of all moments of a distribution) likely never occurs (Nason 2006). Even then, though, it is not easy to detect when the assumption of WSS (WSSH) might be valid. Most approaches toward determining stationarity (homogeneity) that are found in the literature depend upon developing a statistical model of the data. However, sometimes one model works for one dataset, but not for other (Nason 2006; Serinaldi et al. 2018). Moreover, all such models make assumptions about the statistical homogeneity data, which may or may not be valid.

In a set of observations, there are random fluctuations superposed on values correlated in time and/or space. Furthermore, even though every set of observations has one average value, it may be an average over more than one "global" mean value each contributing to the observations. Here the word "global" is used to imply over an entire set of observations as opposed to local apparent mean values arising from correlated fluctuations around the global mean values. As discussed in Jameson et al. (2018), these components can be separated. Hence, an alternative approach for determining whether or not a set of data is likely WSS (WSSH) or not can be defined only using the available data without assumptions, as recently presented by Jameson et al. (2018); namely, does the data have more than one global mean value? And second, does the variance of the data change within the set of observations? First, changes in the variance can be detected using the technique described in Anderson and Kostinski $(2010,2011)$ and described further below. Second, the appearance of more than one global mean value can be determined using a Bayesian inversion technique (Jameson 2015; Jameson and Heymsfield 2013, 2014; Jameson et al. 2018; Jameson 2007). Hence, the data can be used directly to estimate whether or not a set of observations is likely WSS (WSSH) because either there is more than one global mean value and/or the variance of the data is changing in time or space. Consequently, an index of statistical heterogeneity (IXH) has been developed (Jameson et al. 2018) for evaluating whether or not a time series of rainfall measurements may be considered to be temporally statistical stationary or spatially statistically homogeneous.

There is also the alternative approach of simply determining whether or not the calculated correlation function and the DFT-calculated variance spectrum form a Fourier transform pair. This is not necessarily as trivial as it sounds because of fluctuations and noise. However, this approach as well as that using IXH are both investigated below. The IXH approach for identifying WSS (WSSH) at least has an accounting for noise. Nevertheless, in both cases the source of deviations from WSS (WSSH) caused by the correlation functions are the same, but they are approached from different directions as discussed further below.

Moreover, it is not obvious when or how significant deviations from statistical homogeneity become important. In this work we begin to address this question in two ways. First it is argued and shown that when the data are statistically heterogeneous, the Fourier transform of the autocorrelation function cannot yield a true power (variance) spectrum so that it should not be used to scale the variability of rain except for this one specific set of observations. Second, it will be shown that this transform is then dependent on the initial time or location thus negating any potential generality. Third, it will be shown how the magnitudes of Fourier transforms of the autocorrelation functions are altered when there are regions within the sampling domain having different global mean values. The subsequent section after that will consider detailed numerical examples demonstrating these findings. Examples using real data are included in the appendix.

\section{Background}

\section{a. Setting the stage}

There are two important characteristics that a Fourier transform of the autocorrelation function must possess in order to be considered a power spectrum useful for scaling. The first is that it 1) does not depend upon position either in time or space. This makes sense with regard to scaling because if it were not true then it would lack any universal applicability so that the scaling would become different at every location and time. The second is that 2) the transform pair between the autocorrelation function and the power spectrum remain valid. As we will demonstrate later, this can only be true when the variance is constant throughout and there is only one global mean value in the set of observations. As an aside, it should be remembered, however, that statistical mixtures have just one peak in the distribution of mean values and constant variance even though they can have multiple components contributing randomly in a spatially and/or temporally integrated (nonlocalized) manner to the observations. Consequently, statistical mixtures are WSS (WSSH). Let us now first consider item 1. In doing so, only one simulation for each set of conditions is considered since the objective of this study is to highlight the factors affecting the resulting spectra when conditions are not WSS rather than to present a study of rain itself.

Real rainfall is sometime purported to follow a lognormal distribution (Biondini 1976) based solely upon 
rainfall observations. Although Kedem and Chiu (1987) provide a more theoretical justification, Jameson and Kostinski (1999) argue, using the central limit theorem, that since rainfall is additive rather than multiplicative [as implied by a lognormal distribution used by Seed (2003) for describing observations], rainfall more likely follows a gamma distribution, which is found to work quite well for many observations. There are, of course, many other much more complex multifractal models of rain as well, which may or may not be valid (e.g., Serinaldi 2010). However, since this work is not really a study of rain, per se, for simplicity, we just use a Gaussian distribution. We also only focus on temporal data realizing that the results also have an analogous meaning in space. In Fig. 1 two joined regions of Gaussian-distributed rainfall having well-defined means and variances are illustrated. Each region has a different decreasing exponential correlation length $\left(X_{L}\right)$ or time to decorrelation (I), different mean value $(\mu)$, and different variances $\left(\sigma^{2}\right)$. The first is characterized by $\mathfrak{I}_{1}=29, \mu=10$, and $\sigma^{2}=2.5$, while the second has a shorter decorrelation length, larger rainfall rate, and larger intrinsic variance, $\Im_{2}=5, \mu=15$, and $\sigma^{2}=6$. The red box represents the sampling domain with the leftmost edge located at a starting time of $T_{0}=$ $400 \mathrm{~min}$ as discussed further below.

Let us consider some time series of a statistically homogeneous set of measurements represented by the function, $f(t)=\delta(t)+\mu$, where $\mu$ is the mean and $\delta$ is the zero-mean random deviation around that mean. Now suppose that the observations are drawn from a set of $N$ distinct homogeneous components denoting the $i$ th component by $f_{i}(t)$ each having their own mean value $\mu_{i}$ and deviation $\delta_{i}(t)$. Also suppose that this component contributes fraction $F_{i}$ to the total observations. When a correlation is computed, the data are assumed to be statistically homogeneous having a single global mean value $\mu_{g}$ over the entire PDF $\mu_{g}=\sum F_{i} \mu_{i}$ where the summation is over all the contributing components. This mean must then be subtracted before computing the correlation function. For an infinite real value domain, the correlation function $\rho$ can be expressed as the convolution, $f * f$. After expanding the terms and noting that the average $\delta_{i}=0$ we then have that the total correlation function $\rho_{T}(\tau)$ would be

$$
\begin{aligned}
\rho_{T}(\tau)= & \left\langle\sum_{i=1}^{N} F_{i}\left[\delta_{i}(t)+c_{i}\right] * \sum_{i=1}^{N} F_{i}\left[\delta_{i}(t+\tau)+c_{i}\right]\right\rangle \\
= & \sum_{i=1}^{N} F_{i}^{2} \rho_{i}(\tau)+2 \sum_{i=1}^{N-1} \sum_{j=i+1}^{N} F_{i} F_{j} \operatorname{cov}\left(\delta_{i}, \delta_{j}\right) \\
& +2 \sum_{i=1}^{N-1} \sum_{j=i+1}^{N} F_{i} F_{j} c_{i} c_{j}+\sum_{i=1}^{N} F_{i}^{2} c_{i}^{2}
\end{aligned}
$$

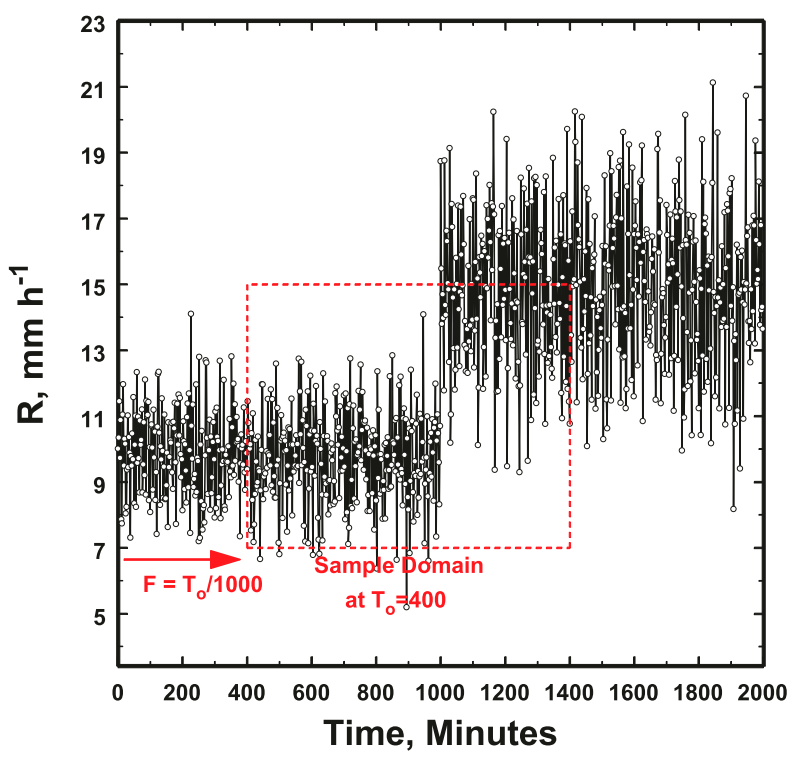

FIG. 1. Simulated data for two joined Gaussian distribution of rainfall rates having different mean values as discussed in the text. The red box is the 1000-min sampling domain that is moved across the entire set of data with the origin as indicated. As the sampling domain moves in time, the correlation functions and their Fourier transforms also change.

where $<>$ denotes averaging and after defining $c_{i}=$ $\mu_{i}-\mu_{g} ; \rho_{i}$ is the autocorrelation function for the $i$ th component, $\tau$ is the lag time, and the subscript $T$ denotes total value. The correlation function then consists of a WSS term [the first summation on the right-hand side of (3)], a convolution term [the second summation on the right-hand side of (3)], and mean values terms [the last two terms on the right-hand side of (3)].

If one were to blindly take this correlation functions and perform a Fourier transform in accordance with the Wiener-Khintchine theorem one would then have

$$
\begin{aligned}
S_{T}(\omega)= & \sum_{i=1}^{N} F_{i}^{2} S_{i}(\omega)+2 \sum_{i=1}^{N-1} \sum_{j=i+1}^{N} F_{i} F_{j} \widetilde{\Re}\left[\operatorname{cov}\left(\delta_{i}, \delta_{j}\right)\right] \\
& +\delta(\omega)\left(2 \sum_{i=1}^{N-1} \sum_{j=i+1}^{N} F_{i} c_{i} F_{j} c_{j}+\sum_{i=1}^{N} F_{i}^{2} c_{i}^{2}\right),
\end{aligned}
$$

where $\mathfrak{\wp}$ represents the Fourier transform, $S_{T}$ is the total spectrum, and $\delta(\omega)$ represents the Dirac delta function. Since each component is WSS, the $S_{i}$ are the power spectra for the distinct separate components, and cov is the covariance function. If the time-series components were statistically independent such that the mean values of $\operatorname{cov}\left(\delta_{i}, \delta_{j}\right)$ would be zero, the Fourier transform $\mathfrak{F}\left[\operatorname{cov}\left(\delta_{i}, \delta_{j}\right)\right]$ need not be null in general unless the fluctuations were nearly statistically independent. If that were the case, then $S_{T}$ would be 
$S_{T}(\omega)=\sum_{i=1}^{N} F_{i}^{2} S_{i}(\omega)+\delta(\omega) \times \mathrm{DC}$ while the inverse Fourier transform of $S_{T}$ would just be $\rho_{T}(\tau)=$ $\sum_{i=1}^{N} F_{i}^{2} \rho_{i}(\tau)$ if the spike at $\omega=0$ [the so-called direct current (DC) with no frequency spike] were filtered out first. Under these circumstances the requirements for applying the Wiener-Khintchine theorem would be satisfied. However, in general these conditions are unlikely to be satisfied. Moreover, there is also another fundamental reason why $S_{T}$ cannot be a power spectrum as discussed in a moment.

Before proceeding, however, consider four cases, three of which are to be illustrated in a later section. In case 1 , consider a WSS system of one component. In that case, $c_{1}$ and $F_{2}$ both equal zero so that (3) and (4) simplify to identities as they should. In case 2 , suppose we have a system having two distinct components with a constant mean value for both components having the same correlation functions (i.e., the variances are distributed the same across frequencies) but each component is associated with a different total variance (i.e., $\delta_{1} \neq \delta_{2}$ mostly at longer lags) but with $F_{1}=F_{2}=$ 1/2. In that case, $c_{1}=c_{2}=0$ so that (3) becomes $\rho_{T}(\tau)=1 / 2 \rho(\tau)+1 / 2 \operatorname{cov}\left(\delta_{1}, \delta_{2}\right)$ so that (4) simplifies to $S_{T}(\omega)=1 / 2 S(\omega)+1 / 2 \mathfrak{r}\left[\operatorname{cov}\left(\delta_{1}, \delta_{2}\right)\right]$. Consequently, the Fourier transform of $\rho_{T}(\tau) \neq S(\omega)$ and the inverse Fourier transform of $S_{T}(\omega) \neq \rho(\tau)$. Hence, this case is not WSS. In case 3 , we take a two-component system having the same mean value but different correlation functions so that the variance (fluctuation) spectra are different. The results will be like those for case 2 .

Sometimes, however, in a set of observations, the rainfall can still come from more than one distribution function yet remain statistically homogeneous. While each component makes a contribution to the resulting distribution function $p(R)$ at each $R$, the distribution of these mean values and variance does not depend upon origin; that is, the same distribution of mean values and variance are applicable everywhere, just like a well-mixed cake batter. The $p(R)$ is then referred to as a statistical mixture (e.g., Feller 2009, p. 55). This contrasts with statistical heterogeneity in which the distribution of mean values and variance does depend upon location (origin) just like a cake batter awaiting mixing.

In case 4 , we consider a two-component system with different mean values but the same variance throughout, that is, just one correlation function. This is often referred to as a homogeneous statistical mixture. Then the last two terms in (3) [and, therefore, in (4)] combine (because the covariance is unity) to be zero by the definition of $\mu_{g}$ while the first two terms reduce to $\rho_{T}(\tau)=$ $\rho(\tau)$ so that $S_{T}(\omega)=S(\omega)$. Consequently, $\rho_{T}(\tau)$ and $S_{T}(\omega)$ form a Fourier transform pair; that is, the statistical mixture is WSS. For other more general cases, the

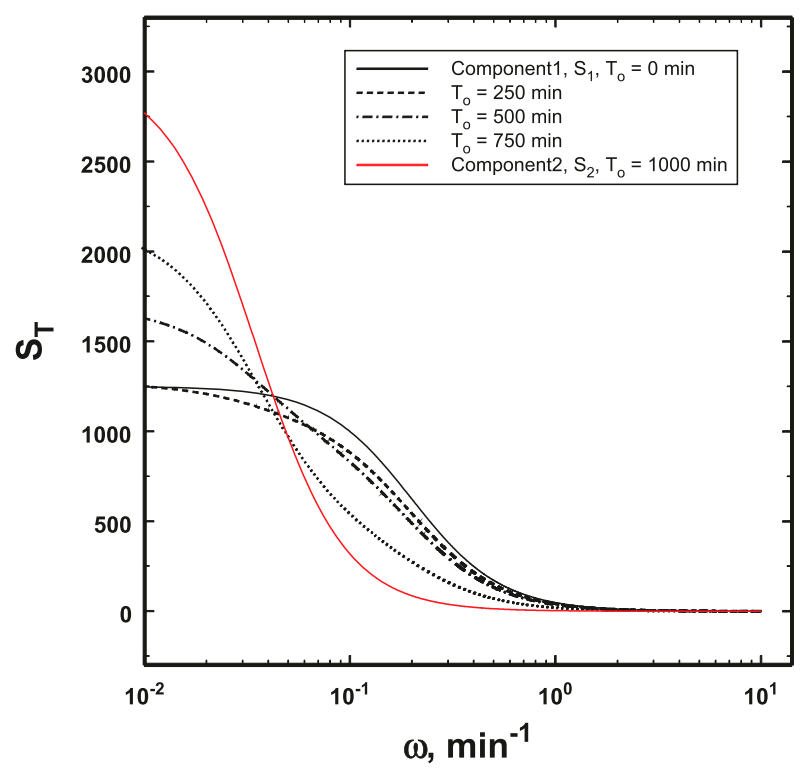

FIG. 2. The total power spectra $\left(S_{T}\right)$ as functions of the starting position of the sampling domain as it moves across the entire domain in Fig. 1. Note that at $T_{0}=0 \mathrm{~min}$, the spectrum is only that of component $1, S_{1}$, while at $T_{0}=1000 \mathrm{~min}, S_{T}=S_{2}$. At the two ends, the data are statistically homogeneous, while statistical heterogeneity prevails in between those extremes. This produces power spectra, which change with start time inconsistent with statistical homogeneity.

relations (3) and (4) then describe what happens when there are multiple distinct contributing components having different mean values and correlation functions. We will consider several examples in the next section.

However, to start with, consider a simplified system in Fig. 1 described previously. Each component was then each exponentially correlated using the method of Fox et al. (1988) with decorrelation lengths of 29 and $5 \mathrm{~min}$, respectively. This methodology has been used throughout this work.

Hence, as Fig. 2 illustrates for the data in Fig. 1, the final total spectrum $S_{T}$ depends upon starting time. However, power spectra must be independent of starting time if they are to represent useful and general scaling relations as pointed out by others (Dechant and Lutz 2015; Leibovich and Barkai 2017). Therefore, because of this time dependence, $S_{T}$ cannot represent a power spectrum.

This can also be illustrated by looking (Fig. 3) at the total variance derived by integrating $S_{T}$ over all the different spectra for the different $F$. Obviously the total variance also depends upon the starting time, which is not consistent with statistical stationarity. Equivalently, by replacing time with distance, the total variance would also depend upon the location so that all these results are also inconsistent with statistical spatial homogeneity. Furthermore, the covariance becomes a 
significant component that will distort any inverse Fourier transform attempting to extract a correlation function. Thus, WSS statistical stationarity is required if the Fourier transforms of correlation functions are to be useful power (variance) spectra. Hence, the presence of more than one component having different fluctuation spectra in a set of observations destroys any possibility of $S_{T}$ being a power spectrum having any generality.

It becomes essential, then, to determine whether or not a set of observations possesses any potential generality by determining whether or not it is statistically homogeneous in the wide sense. One such approach developed by Jameson et al. (2018) is reviewed briefly in the next section and illustrated using the data presented in Fig. 1. We will then look more carefully at several different cases as a representative initiation into the exploration of the sensitivity of this scheme to different mean values and to changing variances. Results from the direct FFT approach are also discussed.

\section{b. The definition and an application of the statistical heterogeneity index}

We begin by considering one definition of an IXH. Many such definitions are possible, of course, but this one is chosen because of its dependence on the number of detected contributing components and because it depends upon changes in the variance of a variable, each a requirement for statistical heterogeneity. Specifically, we use the definition in Jameson et al. (2018):

$$
\mathrm{IXH}=\left\{\frac{H\left(\frac{\mathrm{RD}_{\alpha}}{1.5}-1\right)\left(\frac{\mathrm{RD}_{\alpha}}{1.5}-1\right)+\left(N_{c}-1\right)}{2}\right\}
$$

Here, $H$ is the Heaviside unit step function, $\mathrm{RD}_{\alpha}$ is the relative dispersion of the record-counting parameter $\alpha$ used to detect changes in the variance as discussed below, and $N_{c}$ is the number of detected contributing components in a series of observations, also explained below, and is always $\geq 1$. As discussed more completely in Jameson et al. (2018) statistical heterogeneity is increasingly likely as IXH increases beyond zero. However, statistical mixtures can also be identified when $N_{c} \geq 1$ but $\mathrm{RD}_{\alpha} \approx 0$.

The IXH is a combination of observations of two factors. First, it accounts for the presence of more than one component $\left(N_{c}\right)$ in the distribution of mean values that violates one of the requirements for the observations to be WSS (WSSH). The second factor is the detection of changes in the variance of the variable as

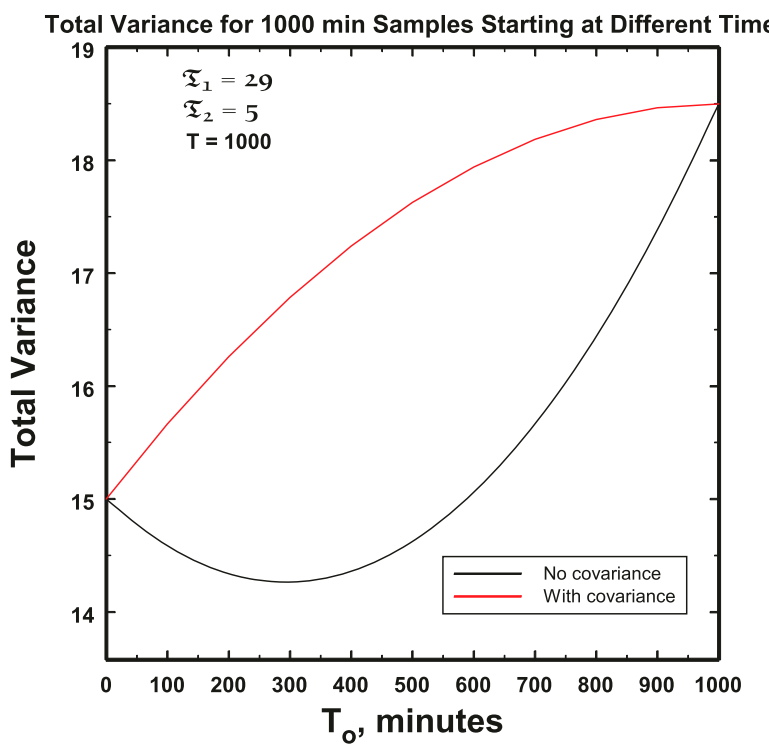

FIG. 3. The total variance integrated over the spectra such as in Fig. 2 showing that the total variance changes with starting time and that the Fourier transform of the covariance function of the observed correlation function contributes significantly to the total variance. For statistically homogeneous data, there is no covariance function and the total variance is independent of starting time or spatial position.

measured by record counting $(\alpha)$ discussed in greater detail below. Since $\alpha$ is a random variable, it will exhibit fluctuations so that a standard for statistical meaningfulness must be established. This is done using a relative dispersion defined here to be $\mathrm{RD}_{\alpha}=\alpha / \sigma_{\alpha}$. Consequently, assuming a normal distribution (as observed in data), about $90 \%$ of the statistical fluctuations of $\alpha$ will produce a relative dispersion $\mathrm{RD}_{\alpha}=\alpha / \sigma_{\alpha} \leq 1.5$ so that only those values $>1.5$ are considered indicators of statistical heterogeneity. Such a value indicates a statistically meaningful change in the variance in violation of the second requirement for WSS (WSSH).

To elaborate further, a Bayesian inversion method has been developed for estimating the probability distribution of the mean values, $p(C)$, of a series of observations (Jameson 2007; Jameson and Heymsfield 2013, 2014; Jameson 2015). While developed in greater detail in those references, we start with an assumed distribution such as a Gaussian. We then assume a range of mean values $C$. Each observation can then be associated with each of these mean values at a computed probability. By summing these probabilities over all the observations and after normalization, we then end up with the likely distribution of these mean values $p(C)$. The most likely mean values are those at the peaks in the distributions. As discussed further in Jameson et al. (2018), these peaks can usually be identified using first and second 
derivatives (Arteaga-Falconi et al. 2015; Slodzinski et al. 2013) especially when considered in conjunction with the structure of $p(C)$ itself. These results are not sensitive to correlations because $p(C)$ does not depend upon whether the observed values are clustered or spread out in time or space.

With regard to the variance, $\alpha$ is the summation of the record minima and maxima of the fluctuations in the forward direction minus the record minima and maxima of the fluctuations in the backward direction along, say, a time series (e.g., see Fig. A2a) as discussed in detail in Jameson et al. (2018) for both one- and two-dimensional data. Specifically, $\alpha=\left(N_{H}+N_{L}\right)_{\text {fwd }}-\left(N_{H}+N_{L}\right)_{\text {bwd }}$, where $N_{H}$ are the number of record highs, $N_{L}$ are the number record lows, and the subscripts fwd and bwd denote the forward and backward directions, respectively. If the variance of the variable is constant, $\alpha=0$. Assuming that the effects of correlations and trends have been removed, deviations from the null are indicative of changes in the variance throughout the domain consistent with statistical heterogeneity.

Anderson and Kostinski (2010, 2011) showed that $\alpha$ appears to be approximately normally distributed, and that its variance depends upon the number of records. This was expressed explicitly in Jameson et al. (2018), where it was shown that the standard deviation $\sigma_{\alpha}$ for the distribution of $\alpha$ is given by

$$
\sigma_{\alpha}=[4 \ln (N)-4.271]^{1 / 2},
$$

where $N$ is the number of observations as illustrated in Fig. 4. A minimum requirement for WSS (WSSH) is that $N_{c}=1$, that is, that there is only one peak in the distribution of mean values and that $\alpha=0$.

Returning once again to the example in Fig. 1, we now plot $N_{c}, \mathrm{RD}_{\alpha}$, and IXH as functions of the starting time, $T_{0}$ in Fig. 5. Starting with the statistically homogeneous conditions when $T_{0}=0 \mathrm{~min}$ and ending with the statistically homogeneous conditions when $T_{0}=1000 \mathrm{~min}$, we see that as soon as $T_{0}$ reaches $100 \mathrm{~min}$, statistical heterogeneity is detected because $N_{c}$ increases from 1 to 2 while $\mathrm{RD}_{\alpha}$ increases to values $\geq 1.5$. As one might have anticipated, the variability in IXH is due entirely to fluctuations in $\mathrm{RD}_{\alpha}$. Note that for the initial point in Fig. 5, IXH $=0.2$ even though the data are WSS. According to Fig. 4, this should only happen $3 \%$ of the time, but clearly it still can happen. Hence, IXH as defined by (3) is not always going to be fail proof because of statistical fluctuations. When the values of IXH are small, one can always try using the DFT approach to verify the finding, as discussed later.

For completeness, Fig. 6 illustrates the distributions of the mean values deduced using the Bayesian technique

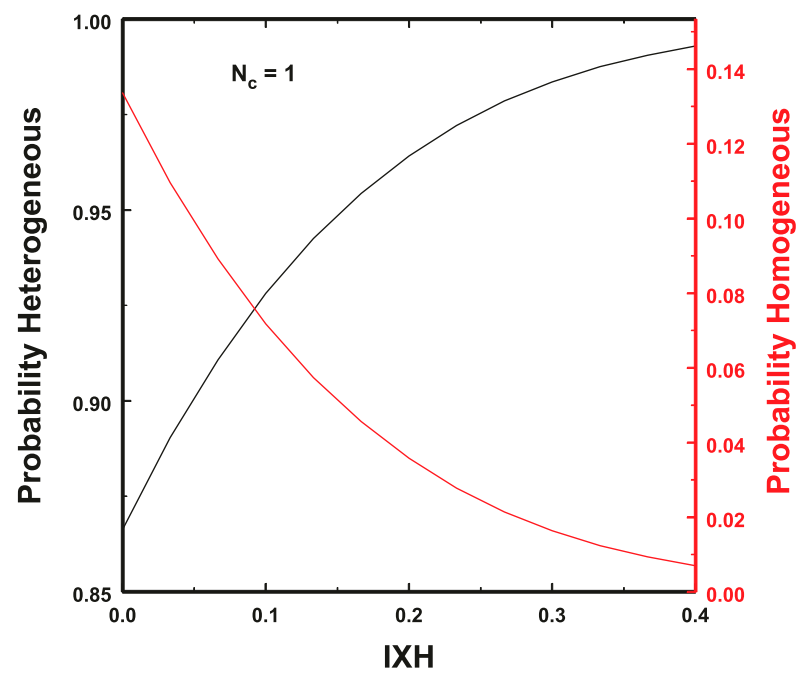

FIG. 4. When the number of detected components is unity $\left(N_{c}=1\right)$, fluctuations in $\alpha$ can make statistically homogeneous data appear to be statistically heterogeneous. The probabilities that the data were really statistically heterogeneous (black line) and the probabilities that the data were really statistically homogeneous (red line) are plotted as functions of the calculated IXH. Hence, as IXH increases, the likelihood that the data are statistically homogeneous decreases.

described above. The $p(C) \mathrm{s}$ at $T_{0}=100,600$, and $900 \mathrm{~min}$ are plotted. The peaks are associated with the most likely values as determined by two methods, namely, where the first derivative is zero (standard calculus) and the second where the second derivative is a negative minimum (Arteaga-Falconi et al. 2015; Slodzinski et al. 2013). The method of detection of each component is also indicated. This illustrates the importance of the second derivative detection when the contribution from a component to the total time series is only $10 \%$ of the time series.

While in this example the contribution of $N_{c}$ to IXH is obvious, it is the variance that produces the fluctuations. These fluctuations depend upon the intrinsic variability of the quantity itself and the variability arising from of how the data are distributed in time or space. We begin to explore these two sensitivities in the next section.

\section{Other examples}

As a reminder, the focus of this work is on exploring the mathematical details of why WSS or WSSH are required for deriving true power spectra often used in scaling studies of rain. This must be done using simulations. However, while Jameson et al. (2018) provide many examples of the application of IXH to real data, for completeness here we also provide an example of the analysis of real data in the appendix for interested readers. 


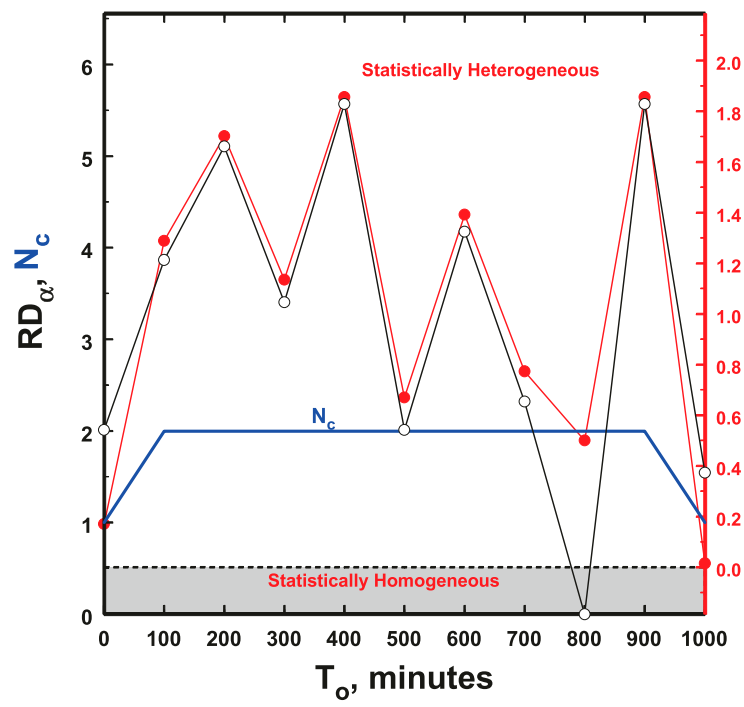

FIG. 5. The changes in the relative dispersion of $\alpha\left(\mathrm{RD}_{\alpha}\right)$ (black), the number of detected components $N_{c}$ (blue), and the resulting IXH (red) plotted as functions of starting time showing that the data are statistically heterogeneous at all starting times except at the ends when the data are statistically homogeneous (stationary).

Again, however, the purpose of this section is to illustrate the effects of statistical heterogeneity, arising for different reasons, on the power spectra as well as on the correlation function. To simplify, we use (3) and (4) but only for an equally divided two-component time series. For comparison, we also compute the spectra and correlation functions for statistical mixtures of the two distinct components in order to highlight the departures of the spectra and correlations from those when conditions are not WSS.

In the first example, the histogram in Fig. 7a does not show anything that suggests a deviation from statistical homogeneity. However, we have two distinct components, each with the same mean value so that $N_{c}=1$, each with the same correlation length $X_{l}=29 \mathrm{~min}$, but with the first and second components having different intrinsic variances of 2.5 and $6 \mathrm{~mm}^{2} \mathrm{~h}^{-2}$, respectively, as discussed above as case 2. As illustrated in Fig. 7b, while each component is statistically homogeneous having $\mathrm{IXH}=0$, when they are joined the data are now statistically heterogeneous, simply because of the change in the variance across the time series.

Moreover, we then compute the respective power spectra as illustrated in Fig. 7c. The $S_{T}$ is the Fourier transform of the observed correlation function for the data in Fig. 7b. This can be compared to the Fourier transform had the data been a true WSS statistical mixture [ i.e., $S_{T}(\omega)=\sum_{i=1}^{2} F_{i}^{2} S_{i}(\omega)$ ], having no cov or constant terms in (3) and (4). Obviously, the differences between the two spectra are significant, illustrating that

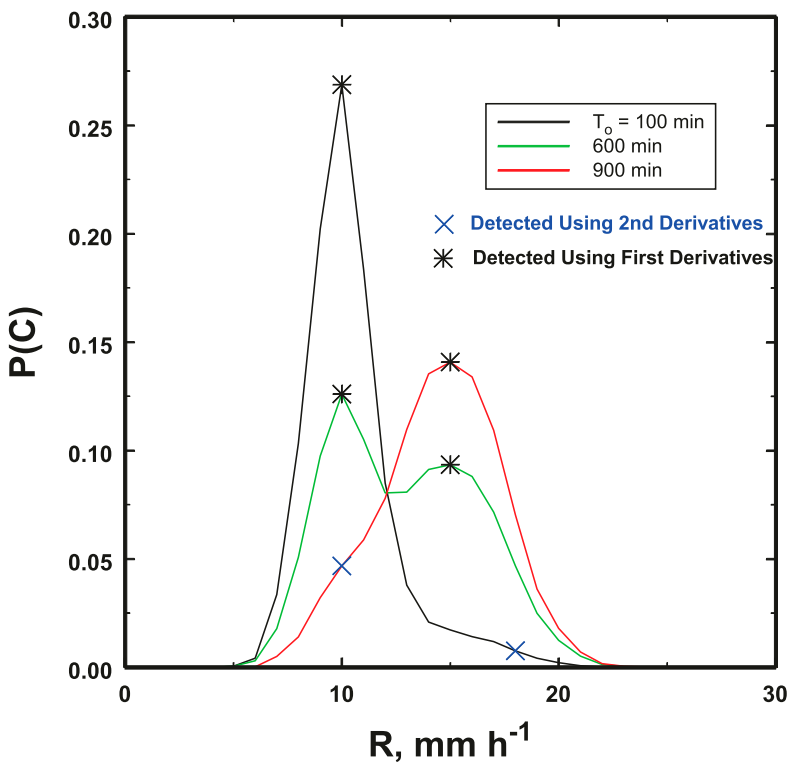

FIG. 6. Distributions of the mean values determined from the Bayesian inversion showing which techniques led to the identification of the peaks associated with distinct contributing components using the first and second derivative approaches explained in the text. Even when the data contained only $10 \%$ of a component, the two peaks could be identified in this example.

something as straightforward as a change in the intrinsic variance as a function of time or distance can profoundly affect the power-spectra characterization of the data. Hence, any scaling power law deduced from $S_{T}$ for the statistically heterogeneous data would be significantly different from that deduced for WSS conditions that would yield a valid scaling.

If we also consider the direct FFT of the data we get the variance spectrum illustrated in green having a power-law fit closer to that for the WSS mixture. While it is tempting to decide that the DFT approach is better, it turns out to be an illusion as discussed next.

The variance spectra differences, in turn, are reflected in deviations between the autocorrelation function calculated directly from the time series (observed, solid black line partially hidden by the dashed green line) and that estimated from the inverse Fourier transform of $S_{T}$ (solid red line) as shown in Fig. $7 \mathrm{~d}$. The observed function can also be subdivided into two components, namely, the WSS contribution (dashed blue line) calculated from $\sum_{i=1}^{N} F_{i}^{2} \rho_{i}(\tau)$ in (3) and the covariance contribution (magenta line) as given in (3) by $\rho_{T}(\tau)-\sum_{i=1}^{N} F_{i}^{2} \rho_{i}(\tau)$. In this example, since there is no residual difference between the component and global mean values $\left(N_{c}=1\right)$, it is the covariance terms in (3) and (4) that produce the deviations in both the observed and, therefore, inverse Fourier transform functions from that corresponding to WSS conditions. This difference 

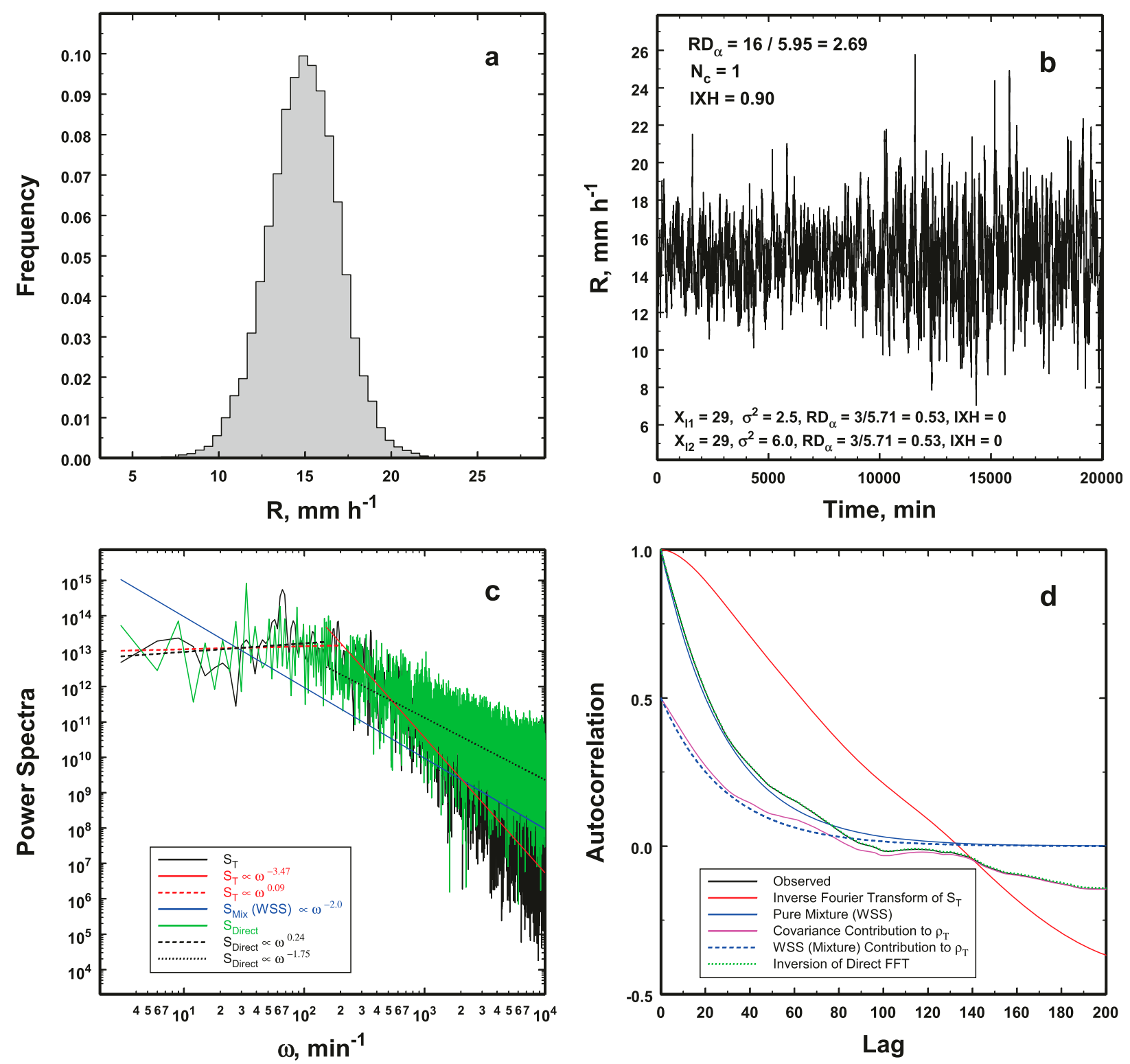

FIG. 7. (a) Simulation of two 10000 Gaussian-distributed rainfall rates having the joint histogram, but (b) joined to form the time series. (c) Only the intrinsic variance for each distribution was changed leading to the differences in the spectra shown here. $S_{T}$ is the total spectrum derived from the Fourier transform of the observed correlation function. The direct transform of the time series yields the green spectrum. The physically meaningful spectrum is given by the mixture (WSS) (blue). The Fourier inversion correlation functions are shown in $(\mathrm{d})$.

mostly appears at lags greater than 80 when short temporal fluctuations play a greater role.

Interestingly the correlation function computed by taking the inverse FFT of the directly computed FFT of the data matches the observed correlation function nearly exactly. However, this does not mean that the DFT and this correlation function form a Fourier transform pair since we already know that were we to take the FFT of the observed correlation function we would get $S_{T}$ in Fig. $7 \mathrm{c}$ that is not equivalent to the green spectrum. Hence, the variance spectrum computed using the DFT and the correlation function calculated from the inverse FFT of the DFT spectrum do not form a Fourier transform pair. However, these results highlight the point that regardless of how the variance spectra are computed, the WienerKhintchine theorem is, indeed, inapplicable in this example.

In the second example (Fig. 8), we again have only one mean value so that $N_{c}$ is again unity, but this time 

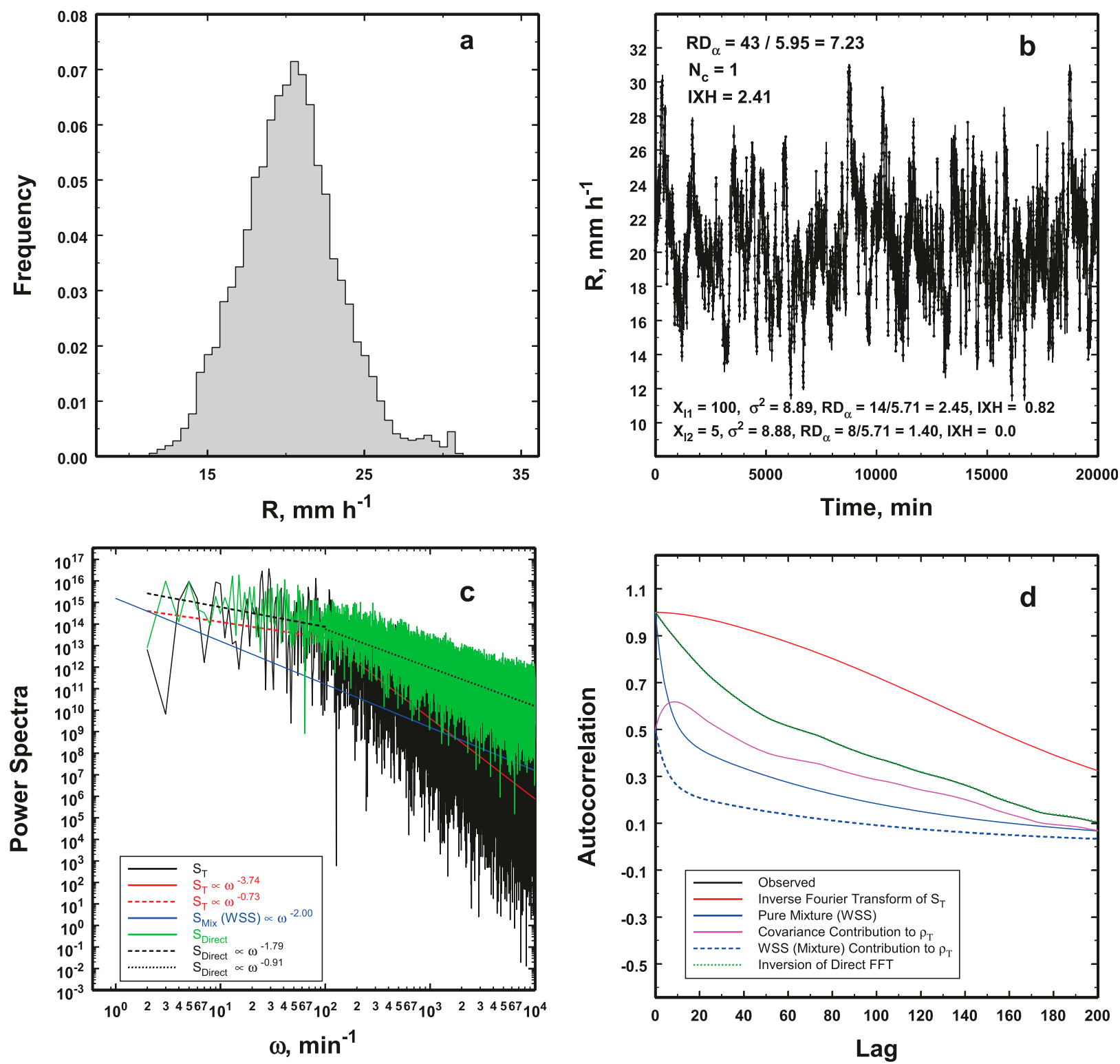

FIG. 8. As in Fig. 7, except that the intrinsic variances remain identical, but the correlation lengths differ leading to different spectra and retrieved correlation functions as discussed further in the text. This shows the sensitivity of statistical heterogeneity to different correlations.

we keep the intrinsic variances the same for the two components, which are identical except for having widely different correlation lengths, $X_{l 1}=5 \mathrm{~min}$ and $X_{l 2}=100 \mathrm{~min}$. This means that the variances are distributed differently over the various frequencies leading to a potentially significant covariance effect in (3) and (4). Again, there is little indication of deviations from statistical homogeneity in the histogram (Fig. 8a) with the possible exception of an enhancement in the tail of larger values. In complete ignorance, such a tail could easily be taken to be a statistical fluke.
Although not readily apparent in the time-series data (Fig. 8b), the first component $C_{1}$ is associated with an IXH $=0.82$ while that for the second component $C_{2}$ is 0 . Yet, when they are combined, the variability in the correlation lengths is sufficient to produce changes in the variance in time of sufficient magnitude so that IXH becomes 2.41, a significant value well into the domain of statistical heterogeneity (see Fig. 4). The reason for this is that while the total variances are the same, they are distributed differently over the different spectral wavelengths. This will, then, affect the covariance terms in (3) and (4). Hence, this heterogeneity is reflected in the 
Fourier transforms in Fig. 8c. As in Fig. 7 the DFT spectrum also differs from $S_{T}$. While the noise parts of the spectra are quite similar, at lower frequencies there are again some significant differences between what would have been expected had the data been statistically homogeneous as compared to the real case of statistical heterogeneity. These differences are also again reflected in the observed and inverse Fourier transform correlation functions as illustrated in Fig. 8d. The covariance term makes the greatest contribution to the observed correlation function at shorter lags (longer wavelengths). As IXH indicates, the data are not suitable for the application of the Wiener-Khintchine theorem. A blind assumption that these data were WSS would have led to significant errors in data scaling compared to truly WSS data. Once again as in Fig. 7d, the correlation function derived from the inverse Fourier transform of the DFT spectrum matches the observed correlation function. Hence the derived correlation function and the DFT spectrum do not form a transform pair because the FFT of the derived correlation function does not match the DFT spectrum.

Finally, we consider an example when the intrinsic variances and the correlation functions are fixed for both components, but the mean values are distinctly different as evident in the histogram in Fig. 9a. As the time series shows (Fig. 9b), it is only the difference in the mean values that indicates statistical heterogeneity leading to IXH $=0.5$. Yet this IXH is significant as demonstrated in the power spectra (Fig. 9c) and in the correlation functions in Fig. 9d. Here the exaggerated differences among the correlation functions arise because the differences between $\mu_{g}$ and each of the component mean values, $\mu$. The difference between the mean values in combination with the covariance contributions clearly overwhelms the WSS contribution to the observed correlation. Of course, no one would have assumed these to be statistically homogeneous data in the first place, but it does illustrate the potential dangers inherent in more subtle situations. Moreover, using the DFT approach does not ameliorate the problem.

\section{Conclusions}

Scaling studies of rainfall are important for the translation of observations and numerical model outputs among all the various scales. For example, given an output from a numerical forecast model on, say, $5-\mathrm{km}$ scales, it would be useful to urban flooding and farm field erosion prevention to know what the corresponding rainfall variability would be on kilometer or smaller dimensions. Likewise, if a network of rain gauges provided observations over a $1 \mathrm{~km} \times 1 \mathrm{~km}$ domain, how could they be incorporated onto the coarser grids of numerical forecast models? Such scaling, however, implies a degree of generality of scaling spectra. Otherwise scaling would only apply to each different situation that then would have to be determined each time, thus defeating scaling as a useful tool with any generality applicable to other observations.

There are several methods for deriving such power spectra including the direct Fourier transform (DFT) of observations and the application of the WienerKhintchine (WK) theorem to the observed correlation function. Regardless of how such spectra are computed, however, all valid power spectra must satisfy the WK theorem. When observations are wide-sense statistically stationary (WSS) or wide-sense spatially statistically homogeneous (WSSH), the Wiener-Khintchine theorem associates an observed autocorrelation function to a scaling variance (power) spectrum as a Fourier transform pair. Because the mean value is a constant under these circumstances, this makes it easy to scale features among all the different observed scales using only an observed correlation function. Thus, it is important to be able to identify WSS conditions so that any scaling has general applicability.

In this work we explore the reasons why WSS (WSSH) is a necessary requirement using controlled simulated data. However, in order to do this, we must also demonstrate that the simulated data are WSS. There are two approaches for doing this. The first is to use the index of statistical heterogeneity (IXH) defined above and in Jameson et al. (2018) for estimating when data may be safely assumed to be WSS. This was accomplished by estimating the number of distinct components (peaks in the distribution) of mean values in the data and by using record-counting techniques to detect changes in the variance of the rain. When either of these quantities exceeds certain limits, IXH exceeds 0 ; the Fourier transform of the observed correlation function should not then be used for general scaling studies. An alternative is to use an observed correlation function and the Wiener-Khintchine theorem to test whether an observed correlation function and its Fourier transform are a Fourier transform pair by inverse transforming the calculated spectrum and comparing those results to the original observed correlation function.

What this study shows, however, is that when conditions are not WSS, the Fourier transform of the observed correlation function not only includes the power spectra of each component, but it also includes the contributions arising from all of cross correlations among all of the contributing components. Moreover, the Fourier transform then becomes "local" in that it also depends upon 

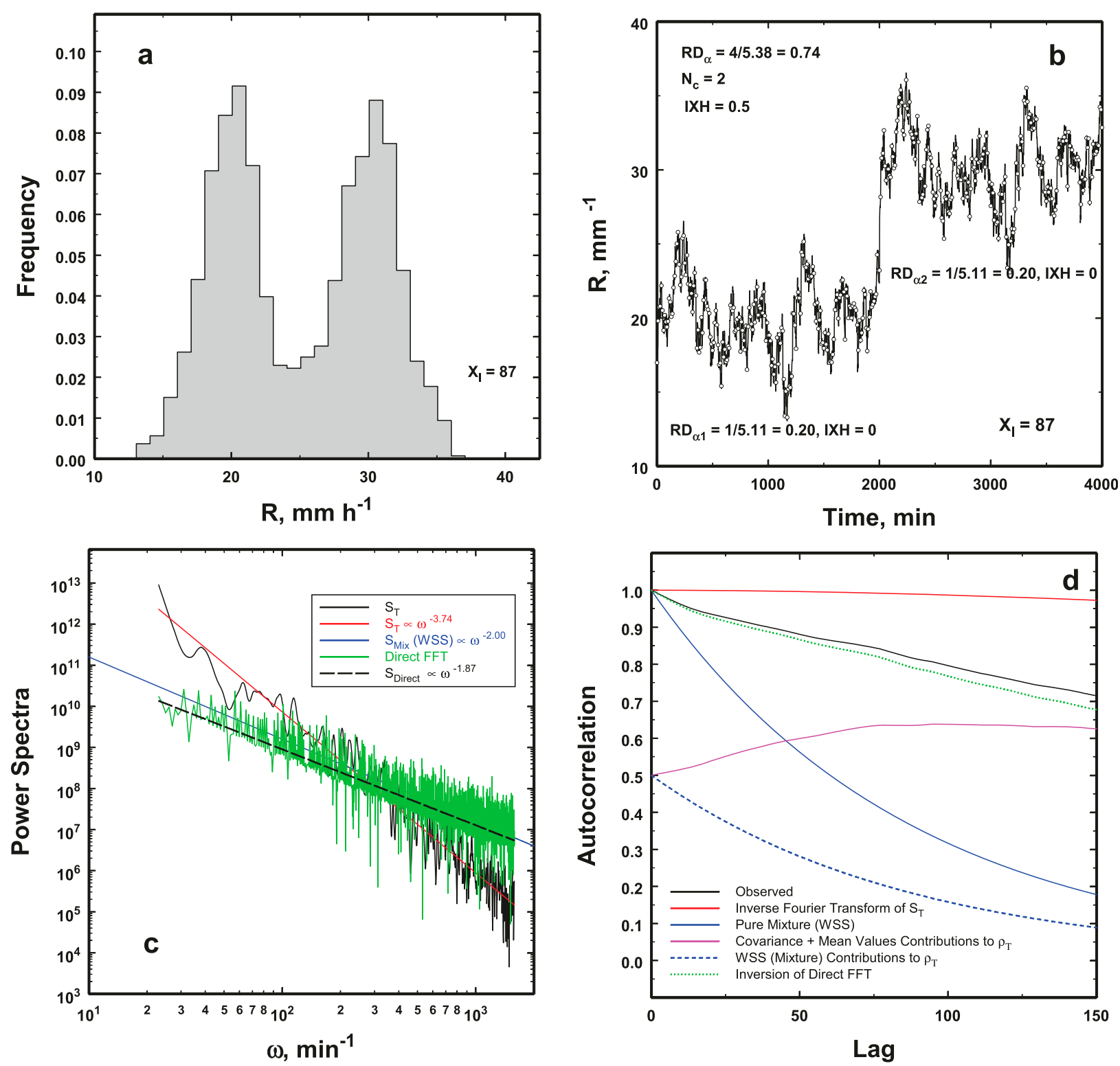

FIG. 9. As in the previous figures, except this time the intrinsic variances and the correlation functions are kept the same, but there are now two different mean values as is evident in (a). The spectra show significantly different powers and the inverse Fourier-derived correlation functions show substantial differences in part because of the significantly different mean values.

the time and location of the particular set of observations. Consequently, there is no longer any generality useful for scaling the rainfall structure for different rainfall measurements.

While it is well known that rainfall data should be WSS (WSSH) in order to use the WK theorem, specific reasons for this are not evident in the meteorological literature. This work fills this void by explaining in specific detail what happens when the Wiener-Khintchine theorem is erroneously applied to simulated statistically heterogeneous rainfall data. In particular, the results from such transformations are compared to those for a statistical mixture of all of the components, which is WSS (WSSH) so that the effects of statistical heterogeneity become readily apparent. It is also shown that the Fourier transform derived using an observed correlation function cannot then be inverse Fourier transformed to again yield the observed correlation function. Hence, in statistically heterogeneous data, the "spectra" and the calculated correlation function do not, then, form a Fourier transform pair as required by the Wiener-Khintchine theorem. This is not surprising, of course. It is also shown that when $N_{c}>1$, IXH is sensitive both to the intrinsic variance of each 


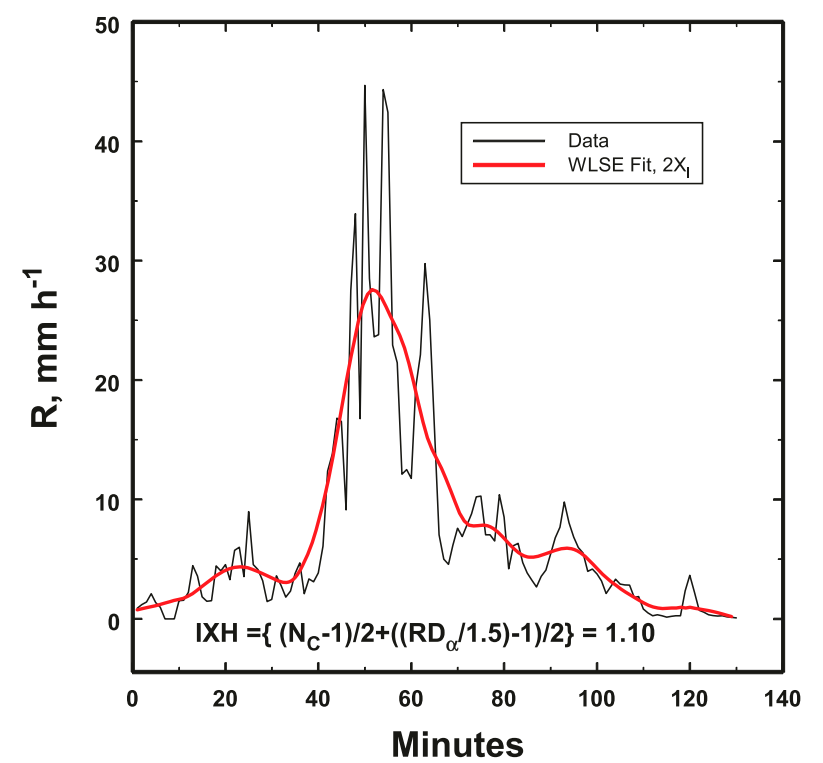

FIG. A1. Observations of the 1-min rainfall rate using a JossWaldvogel disdrometer at the NASA Wallops Island Rainfall Measurement Facility on 3 Mar 2013 during the passage of convective rain associated with a warm front. The red line (as described in the text) represents the estimate of the actual mean value that is affected by correlations in time. The differences between the observations and the means then provides an estimate of the true fluctuations of the rainfall rate used to calculate $\alpha$ as illustrated in Fig. A2a.

of the components contributing to the observations as well as to any differences in the characteristic decorrelation lengths of the correlation functions of each component. Even in cases when there is only one mean value $\left(N_{c}=1\right)$, IXH is still sensitive to the changes in the variance in the time series. IXH, therefore, appears to be a useful tool. While the details of the applicability, of the error rates of IXH and of when deviations from WSS become serious impediments to scaling are yet to be fully determined in future research, the results presented here offer some hope for sorting out useful scaling relations from those that could be misleading.

If it can be shown that the PDF of the components is largely dominated by a single component and that the covariance functions are small, then the power spectrum can be taken to be approximately representative. However, if there is more than one significant component but the covariances are all small (i.e., the fluctuations are statistically independent), then the calculated power spectrum will be the weighted spectrum from each of the components plus an enhanced dc component at $\omega=0$. This later effect should not affect the slopes of the power spectra provided the dc component is excluded in which case the requirements for the WK theorem would be met. The generality of the results,
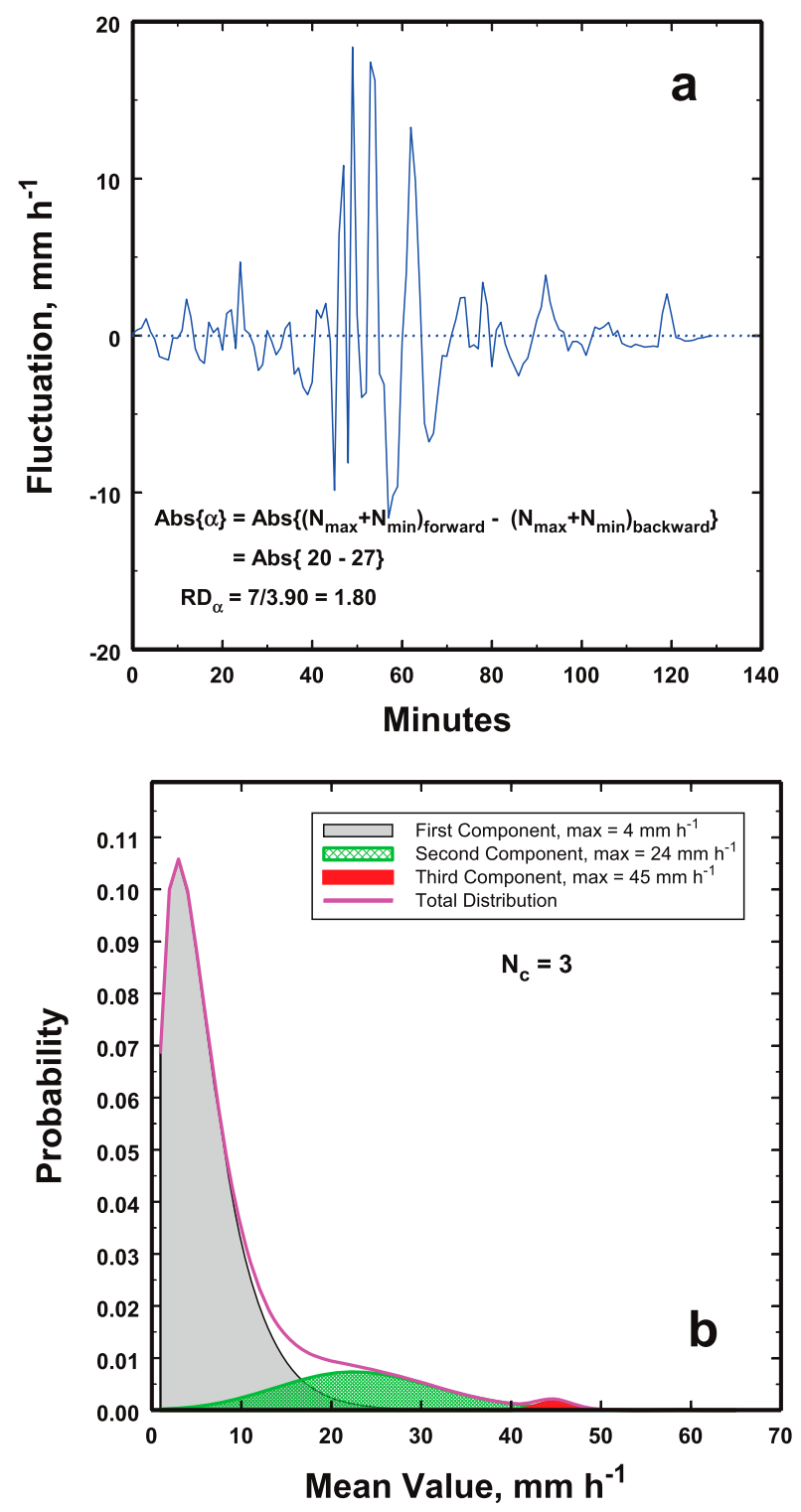

FIG. A2. (a) The plot of the time series of rainfall fluctuations about the mean used in the calculation of $\alpha$ that illustrates the variability of the variance of the rainfall rate over the interval. (b) The distribution of the mean values determined using the Bayesian inversion of the count data as described in the text. The three different gamma fits to the contributing components are illustrated as well.

then, will depend upon the generality of the frequency distribution of the simultaneous occurrence of these components in future measurements as well as nearly statistically independent fluctuations among all the components.

Furthermore, while it is not possible to "correct" for statistical inhomogeneities because they are the reflection of underlying physical-statistical processes, insofar as a statistically heterogeneous set of data may 

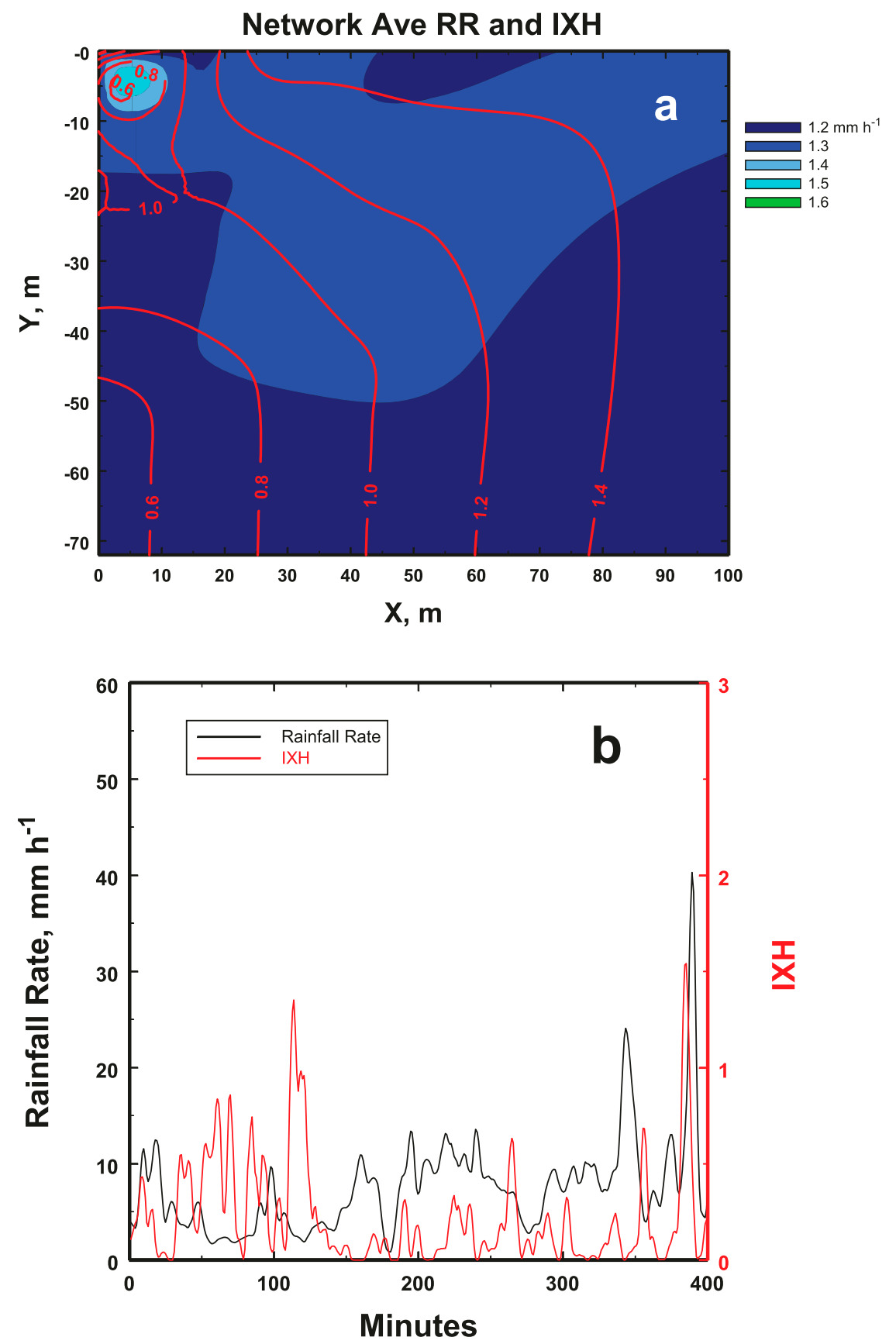

FIG. A3. (a) An example of the spatial variability of the 80-min average rainfall rate, RR, and of the IXH over a small $(100 \mathrm{~m} \times 70 \mathrm{~m})$ network of video disdrometers [described in Jameson et al. (2015)] for a light rainfall event generated by the passage of several different patches of rain having different mean values. Even though the overall rainfall is light, there is still significant IXH because of the different patches of rain. (b) An example of a 5-min smoothed time series over the $121 \mathrm{~cm}^{2}$ surface of a $2 \mathrm{D}$ video disdrometer during a rain event near Charleston, as described further in the text.

consist of the concatenation of several WSS (WSSH) subgroups, it may then be possible, on those occasions, to treat each subgroup independently and then to derive more insight into the scaling. In particular it may, at times, be possible to segment a large set of data into smaller apparently statistically homogeneous segments as illustrated in Jameson et al. (2018). This, again, is the topic for future research. 
Acknowledgments. This work was supported (AJ) by the National Science Foundation (NSF) under Grants AGS1532423 and AGS1823072.

\section{APPENDIX}

\section{An Example of the Analysis of Rain Observations and of Spatial Variability}

The data presented in Fig. A1 are from a JossWaldvogel impact disdrometer. The data [provided by D. Wolff (2013, unpublished data)] were collected at the National Aeronautics and Space Administration rain facility at Wallops Island, Virginia, on 6 March 2013 as a line of convection passed over the facility. As discussed in detail in Jameson et al. (2018), the effects of correlation must be removed in order to calculate the true fluctuation parameter $\alpha$. This is done using a least squares error weighted running average on the data over a length of 2 times the observed decorrelation length. The resulting time series of the fluctuations is plotted in Fig. A2a as is the calculation of the relative dispersion of $\alpha$, namely $\mathrm{RD}_{\alpha}$, that reveals statistically significant changes in the fluctuations of the rainfall rate across the set of observations.

Figure A2a illustrates the fluctuations used to calculate $\alpha$. Figure A2b shows the three components of the distribution of likely mean values resulting from Bayesian inversion of the count data. Gamma distributions are used to fit this distribution since they work quite well, and they are more appropriate (representative of an additive process) to rainfall data than are lognormal distributions (representative of a multiplicative process). These values then combined to yield the observed IXH given in Fig. A1.

As an example illustrating the spatial variability of IXH, Fig. A3a is a plot of the 80 -min average rainfall rate (RR) with overlying contours (red) of IXH over a small network of video disdrometers as described in Jameson et al. (2015). Interestingly, even though the average rainfall rate is light, there is significant IXH because of the passage of several different patches of rain having different mean values during the $80 \mathrm{~min}$.

Finally, an example of a time series of 5-min least squared error weighted average rainfall rate and IXH are plotted in Fig. A3b. IXH was evaluated by sampling the rainfall rate every minute in each $1 \mathrm{~cm}^{2}$ of the $121 \mathrm{~cm}^{2}$ sampling area of a 2D video disdrometer (2DVD) instrument (e.g., see Kruger and Krajewski 2002) near Charleston, South Carolina. The $N_{c}$ and $\alpha$ were then determined over the entire area every minute. Interestingly, $\alpha$ appears to be more strongly related to changes in the rainfall rate than to the rainfall rate itself. This, however, is the subject of ongoing and future research.

\section{REFERENCES}

Anderson, A., and A. Kostinski, 2010: Reversible record breaking and variability: Temperature distributions across the globe. J. Appl. Meteor. Climatol., 49, 1681-1691, https://doi.org/ 10.1175/2010JAMC2407.1.

— and —, 2011: Evolution and distribution of record-breaking high and low monthly mean temperatures. J. Appl. Meteor. Climatol., 50, 1859-1871, https://doi.org/10.1175/JAMC-D-10-05025.1.

Arteaga-Falconi, J., H. A. Osman, and A. E. Saddik, 2015: R-peak detection algorithm based on differentiation. Ninth Int. Symp. on Intelligent Signal Processing, Siena, Italy, IEEE, 157, https://doi.org/10.1109/WISP.2015.7139157.

Berndtsson, R., and J. Niemczynowicz, 1988: Spatial and temporal scales in rainfall analysis-Some aspects and future perspectives. J. Hydrol., 100, 293-313, https://doi.org/10.1016/00221694(88)90189-8.

Biondini, R., 1976: Cloud motion and rainfall statistics. J. Appl. Meteor., 15, 205-224, https://doi.org/10.1175/1520-0450(1976) 015<0205:CMARS $>2.0$. CO;2.

Crane, R. K., 1990: Space-time structure of rain rate fields. J. Geophys. Res., 95, 2011-2020, https://doi.org/10.1029/ JD095iD03p02011.

Dechant, A., and E. Lutz, 2015: Wiener-Khinchin theorem for nonstationary scale-invariant processes. Phys. Rev. Lett., 115, 080603, https://doi.org/10.1103/PhysRevLett.115.080603.

de Lima, M. I. P., W. F. Krajewski, and J. L. M. P. de Lima, 2012: Space-time precipitation from urban scale to global change. Adv. Water Resour., 45, 1, https://doi.org/10.1016/ j.advwatres.2012.06.001.

Feller, W., 2009: An Introduction to Probability Theory and Its Applications. Vol. 2. Wiley, 669 pp.

Fox, R. F., I. R. Gatland, R. Roy, and G. Vemuri, 1988: Fast, accurate algorithm for numerical simulation of exponentially correlated colored noise. Phys. Rev., 38A, 5938-5940, https:// doi.org/10.1103/PhysRevA.38.5938.

Gires, A., I. Tchiguirinskaia, D. Schertzer, A. Schellart, A. Berne, and S. Lovejoy, 2014: Influence of small scale rainfall variability on standard comparison tools between radar and rain gauge data. Atmos. Res., 138, 125-138, https://doi.org/10.1016/ j.atmosres.2013.11.008.

Jameson, A. R., 2007: A new characterization of rain and clouds: Results from a statistical inversion of count data. J. Atmos. Sci., 64, 2012-2028, https://doi.org/10.1175/JAS3950.1.

_ 2015: A Bayesian method for upsizing single disdrometer drop size counts for rain physics studies and areal applications. IEEE Trans. Geosci. Remote Sens., 53, 335-343, https:// doi.org/10.1109/TGRS.2014.2322092.

, 2017: Spatial and temporal network sampling effects on the correlation and variance structures of rain observations. $J$. Hydrometeor., 18, 187-196, https://doi.org/10.1175/JHM-D-16-0129.1. , and A. B. Kostinski, 1999: Fluctuation properties of precipitation. Part V: Distribution of rain rates-Theory and observations in clustered rain. J. Atmos. Sci., 56, 3920-3932, https:// doi.org/10.1175/1520-0469(1999)056<3920:FPOPPV>2.0.CO;2. and A. J. Heymsfield, 2013: A Bayesian approach to upscaling and downscaling of aircraft measurements of ice particle counts and size distributions. J. Appl. Meteor. Climatol., 52, 2075-2088, https://doi.org/10.1175/JAMC-D-12-0301.1. 
and -2014 : Bayesian upscaling of aircraft ice measurements to two-dimensional domains for large-scale applications. Meteor. Atmos. Phys., 123, 93-103, https://doi.org/ 10.1007/s00703-013-0303-3.

—, M. L. Larsen, and A. B. Kostinski, 2015: Disdrometer network observations of finescale spatial-temporal clustering in rain. J. Atmos. Sci., 72, 1648-1666, https://doi.org/10.1175/ JAS-D-14-0136.1.

,-- , and,- 2018 : On the detection of statistical heterogeneity in rain measurements. J. Atmos. Oceanic Technol., 35, 1399-1413, https://doi.org/10.1175/JTECH-D-17-0161.1.

Kedem, B., and L. S. Chiu, 1987: On the lognormality of rain rate. Proc. Natl. Acad. Sci. USA, 84, 901-905, https://doi.org/ 10.1073/pnas.84.4.901.

Khintchine, A., 1934: Korrelationstheorie der stationaren stochastischen Prozesse. Math. Ann., 109, 604-615, https:// doi.org/10.1007/BF01449156.

Kiely, G., and K. Ivanova, 1999: Multifractal analysis of hourly precipitation. Phys. Chem. Earth, 24B, 781-786, https://doi.org/ 10.1016/S1464-1909(99)00080-5.

Koutsoyiannis, D., and A. Montanari, 2015: Negligent killing of scientific concepts: The stationarity case. Hydrol. Sci. J., 60, 1174-1183, https://doi.org/10.1080/02626667.2014.959959.

Krajewski, W. F., G. J. Ciach, and E. Habib, 2003: An analysis of small-scale rainfall variability in different climatic regimes. Hydrol. Sci. J., 48, 151-162, https://doi.org/10.1623/ hysj.48.2.151.44694.

Kruger, A., and W. F. Krajewski, 2002: Two-dimensional video disdrometer: A description. J. Atmos. Oceanic Technol., 19, 602-617, https://doi.org/10.1175/1520-0426(2002)019<0602: TDVDAD $>2.0$.CO;2.

Leibovich, N., and E. Barkai, 2017: Conditional $1 / f^{\alpha}$ noise: From single molecules to macroscopic measurement. Phys. Rev., 96E, 032132, https://doi.org/10.1103/PhysRevE.96.032132.

Matalas, N. C., 2012: Comment on the announced death of stationarity. J. Water Resour. Plann. Manage., 138, 311-312, https://doi.org/10.1061/(ASCE)WR.1943-5452.0000215.

Milly, P. C. D., J. Betancourt, M. Falkenmark, R. M. Hirsch, Z. W. Kundzewicz, D. P. Lettenmaier, and R. J. Stouffer, 2008: Stationarity is dead: Whither water management? Science, 319, 573-574, https://doi.org/10.1126/science.1151915.

Montanari, A., and D. Koutsoyiannis, 2014: Modeling and mitigating natural hazards: Stationarity is immortal! Water Resour. Res., 50, 9748-9756, https://doi.org/10.1002/2014WR016092.
Nason, G. P., 2006: Stationary and non-stationary time series. Statistics in Volcanology, Geological Society, 129-142.

Nerini, D., N. Besic, I. Sideris, U. Germann, and L. Foresti, 2017: A non-stationary stochastic ensemble generator for radar rainfall fields based on the short-space Fourier transform. Hydrol. Earth Syst. Sci., 21, 2777-2797, https://doi.org/10.5194/ hess-21-2777-2017.

Ochoa-Rodriguez, S., and Coauthors, 2015: Impact of spatial and temporal resolution of rainfall inputs on urban hydrodynamic modelling outputs: A multi-catchment investigation. J. Hydrol., 531, 389-407, https://doi.org/10.1016/ j.jhydrol.2015.05.035.

Pegram, G. G. S., and A. N. Clothier, 2001: High resolution spacetime modelling of rainfall: The "string of beads" model. J. Hydrol., 241, 26-41, https://doi.org/10.1016/S0022-1694(00) 00373-5.

Seed, A. W., 2003: A dynamic and spatial scaling approach to advection forecasting. J. Appl. Meteor., 42, 381-388, https://doi.org/ 10.1175/1520-0450(2003)042<0381:ADASSA > 2.0.CO;2.

Serinaldi, F., 2010: Multifractality, imperfect scaling and hydrological properties of rainfall time series simulated by continuous universal multifractal and discrete random cascade models. Nonlinear Processes Geophys., 17, 697-714, https:// doi.org/10.5194/npg-17-697-2010.

_ C. C. Kilsby, and F. Lombardo, 2018: Untenable nonstationarity: An assessment of the fitness for purpose of trend tests in hydrology. Adv. Water Resour., 111, 132-155, https:// doi.org/10.1016/j.advwatres.2017.10.015.

Sinclair, S., and G. G. S. Pegram, 2005: Empirical mode decomposition in 2-D space and time: A tool for space-time rainfall analysis and nowcasting. Hydrol. Earth Syst. Sci., 9, 127-137, https://doi.org/10.5194/hess-9-127-2005.

Slodzinski, R., L. Hildebrand, and W. Vautz, 2013: Peak detection algorithm based on second derivative properties for two dimensional ion mobility spectrometry signals. Integration of Practice-Oriented Knowledge Technology: Trends and Prospectives, M. Fathi, Ed., Springer, 341-354, http://link.springer.com/10.1007/978-3-642-34471-8_28.

Wiener, N., 1930: Generalized harmonic analysis. Acta Math., 55, 117-258, https://doi.org/10.1007/BF02546511.

— D. Jerison, I. M. Singer, and D. W. Stroock, Eds., 1997: The Legacy of Norbert Wiener: A Centennial Symposium in Honor of the 100th Anniversary of Norbert Wiener's Birth. American Mathematical Society, $405 \mathrm{pp}$. 